\title{
Building a Repository to Support Research on Emerging Pathogens: How to Use the Experience of the Centre for AIDS Reagents ${ }^{\dagger}$
}

\author{
Yann Le Duff *, Sarah Gilbert and Neil Almond \\ National Institute of Biological Standards and Control, Hertfordshire SG13, UK; \\ sarah.gilbert@nibsc.org (S.G.); neil.almond@nibsc.org (N.A.) \\ * Correspondence: yann.leduff@nibsc.org \\ + Presented at the 5th African Conference on Emerging Infectious Diseases, Abuja, Nigeria, 7-9 August 2019. \\ Published: 8 April 2020
}

\begin{abstract}
The increasing health threat arising from emerging infectious disease requires an urgent global response. The accelerated development of vaccines, diagnostics and treatments requires facilitated access to and timely transfer of pertinent research reagents between scientists, especially those from low- and middle-income countries (LMICs) which are frequently the worst affected. To meet this global need, we propose to establish a Centre for Emerging Disease Research Reagents (CEDRR) which will support research on those emerging diseases that pose the greatest threat. This initiative will be based on the model of the Centre for AIDS Reagents, a not-for-profit repository based at the National Institute for Biological Standards and Control (NIBSC), UK, which has been providing a sustained HIV research reagent resource to scientists worldwide for 30 years. CEDRR will encourage leading scientists to donate research materials under an established transfer agreement, that will enable onward supply of samples to requesting laboratories in a manner that protects intellectual property. In addition, we will prepare and commission novel research materials including non-infectious subgenomic clones, recombinant proteins, peptides and antibodies. All the reagents will be characterized and provided individually or as all-in-one packages for specific assays along with detailed data sheets and corresponding standard operating procedures. They will be available globally and access will be prioritized with the objective to help build research capacity in LMICs. In order to provide a service that will meet the scientific community need, CEDRR will actively participate in existing networks and consortia on emerging pathogens, provide frequent newsletters and held regular meetings to discuss reagent requirements. By building an infrastructure that will provide quality research reagents to scientists worldwide, CEDRR hopes to speed up the development of much needed vaccines, diagnostics and treatments to fight emerging pathogens.
\end{abstract}

Keywords: repository; research reagent; emerging pathogens; LMICs

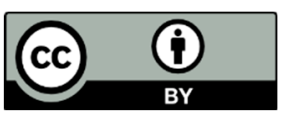

(C) 2020 by the authors. Licensee MDPI, Basel, Switzerland. This article is an open access article distributed under the terms and conditions of the Creative Commons Attribution (CC BY) license (http://creativecommons.org/licenses/by/4.0/). 